\title{
Effects of Pterostilbene on the Activation of Nuclear Factor Erythroid 2-Related Factor 2 Pathway During in vitro Maturation of Mouse Oocytes
}

\author{
Obaid Ullah $^{1 \dagger}$, Li Zhongshu ${ }^{1 \dagger}$, Ihsan Ali ${ }^{1}$, Lijie Xu ${ }^{1}$, Haixing Liu ${ }^{1} \&$ Nanzhu Fang ${ }^{1}$ \\ ${ }^{1}$ Department of Animal Science, Agricultural College, Yanbian University, Yanji, Jilin, China \\ Correspondece: Nanzhu Fang, Laboratory of Animal Genetic Breeding and Reproduction, Department of Animal \\ Science, Agricultural College, Yanbian University, Yanji, Jilin 133002, China. Tel: 86-433-243-5525. Fax: \\ 86-433-243-5600. E-mail: nzfang@ybu.edu.cn
}

\author{
Received: April 8, 2018 \\ Accepted: May 15, $2018 \quad$ Online Published: June 15, 2018 \\ doi:10.5539/jas.v10n7p35 \\ URL: https://doi.org/10.5539/jas.v10n7p35 \\ ${ }^{\dagger}$ These authors contributed equally to this study.
The research is financed by the National Natural Science Foundation of China (Project No. 31360546).
}

\begin{abstract}
Pterostilbene (PTS) is a natural polyphonic compound known to have biological activities, such as antioxidant and anticancer effects. This study was designed to regulate the effect of pterostilbene on the in vitro maturation (IVM) of mouse oocytes denuded of the cumulus (DOs). Different concentration of PTS was added to IVM media with immature DOs. After maturation, meiosis II (MII) stage rates oocytes, Measurement of reactive oxygen species (ROS) and glutathione (GSH) levels, activation of the Nuclear Factor Erythroid 2 like 2 (NFE2L2) pathway and apoptotic expression of BCL2 family in MII oocytes were determined. Our results showed that: PTS significantly increased the MII rate of DOs $(P<0.05)$. Moreover, PTS decreased the ROS levels in DOs $(P<0.05)$ and increased the GSH levels $(P<0.05)$. Furthermore, PTS addition in DOs significantly increased the protein expression of NFE2L2 in the nucleus and decreased Kelch-like ECH-associated protein1 (KEAP1). PTS significantly increased the antioxidant enzyme expression of catalase (CAT), heme oxygenase1 (HMOX1), and superoxide dismutase (SOD). In addition, PTS lowered the protein expression of apoptotic Bcl-2-associated X protein (BAX) and increased the protein expression of anti-apoptotic B-cell lymphoma2 (BCL2) as well as PTS treatment significantly increased the gene expression of BCL2 and reduced the expression of apoptotic BAX in matured DOs. These results indicated that pterostilbene significantly improved the IVM quality matured of DOs and activate NFE2L2-Keap1 pathway during maturation of oocytes.
\end{abstract}

Keywords: pterostilbene, mouse oocytes, in vitro maturation, nuclear factor erythroid 2 like 2 (NFE2L2), Kelch-like ECH-associated protein1 (KEAP1)

\section{Introduction}

Phytoalexins are antimicrobial and antioxidative substances synthesis from grapes and blueberries are utilized widely as a major constituent for nutraceuticals (Kasiotis, Pratsinis, Kletsas, \& Haroutounian, 2013). There are various research studies which have been narrated different biological activates of stilbene scaffolds (Rossi et al., 2012; Wang et al., 2016). In the biological system, the stilbene activates are mostly polyhydroxylated molecules that carry the ability to unify the hydrogen bonds and to create oxidative electrophilic molecules (Stivala et al., 2001).

Pterostilbene (PTS) is stilbene compound that has phytoalexins properties thus protect the plant from external and internal stress (Alessandro, Marco, di Osti, \& Cesari, 2000). PTS was mostly found in blueberries and grapes but it was first discovered in tree wood, Pterocarpus marsupium (Alessandro et al., 2000). PTS is a dimethylated analog of resveratrol but it is more active due to its natural phytochemical verities of bioactivity, which include protection against oxidative stress, inflammation, apoptosis, and aging effects (Wang, $\mathrm{Yu}, \& \mathrm{Xu}$, 2007). Through natural compounds, nuclear factor erythroid 2-related factor 2 (NFE2L2) activation is encouraging way to protect the cells from oxidative stress, such as resveratrol (Ungvari et al., 2010), piceatannol 
(Lee et al., 2010), epigallocatechin-3-gallate (Na et al., 2010), sulforaphane (Jiang et al., 2010). Pterostilbene has also been used to activate NFE2L2 in different studies. As it activates NFE2L2 signaling pathway which increases the expression of the downstream antioxidant enzyme HMOX1 and GRB (Chiou et al., 2011). Bhakkiyalakshmi et al. (2014) stated that pterostilbene has a significant effect on NFE2L2 activation in pancreatic beta cells and increases the downstream target genes of NFE2L2 include HMOX1, SOD, CAT, GPX and also up-regulate anti-apoptotic gene BCL2 expression and downregulate pro-apoptotic gene BAX and caspase- 3 expressions.

NFE2L2 is a transcription factor that regulates intracellular redox balance and antioxidant thus regulates ROS, senescence, and inflammation (Oh \& Jun, 2018). Numerous studies have revealed that activation of NFE2L2 in ethanol-exposed mouse embryos is involved in increased detoxifying and antioxidant enzymes (Harris \& Hansen, 2012). Recently Lin et al. (2018) reported that inhibition of NFE2L2 by brusatol prevents early mouse embryo development by affecting cell cycle progression from G2 to M Phase. The role of NFE2L2 has been investigated in a different cultural environment in bovine embryos that reached blastocyst stage (Gad et al., 2012). However, under suboptimal culture condition in early bovine embryos, NFE2L2-KEAP1 signaling pathway protects the embryo from oxidative stress (Amin et al., 2014). But also, the development of rabbit embryo was promoted by activation of NFE2L2 during induced oxidative stress condition (Mehaisen et al., 2015).

In vitro environment of oocytes, maturation can be affected by oxidative stress due to the manipulation of the oocytes during collection. Thus, it negatively affects the in vitro oocytes maturation, as well as in vitro fertilization in early development. These oxidative stress conditions lead oocytes to apoptosis, fragmentation, and change in oocyte metabolism and gene expression (Ma et al., 2017). Therefore, protection of oocytes against stress condition by activation of NFE2L2 is an approach to improve oocytes maturation under in vitro conditions.

In this study was designed to evaluate PTS effect on (1) denuded oocytes rate (DOs) at metaphase 11 (M11) stage compare to COCs and DOs in control media, (2) ROS and GSH levels, (3) The activation of the NFE2L2 pathway, and (4) The apoptotic expression of BCL2 family in matured oocytes.

\section{Materials and Methods}

\subsection{Chemicals, Reagents and Animal Ethics Statement}

This study was approved in the guidelines for the care and use of animals of the university. In the present study, all chemicals and reagents were obtained from Sigma-Aldrich Chemical Company (St. Louis, MO, USA), unless otherwise stated. Pterostilbene was purchased from Cayman Chemical Michigan USA (Cas no. 537-42-8).

\subsection{Super Ovulation, Immature Oocytes Collection, and in vitro Maturation}

The Kunming mice were selected from the animal experimental center of the university. Mouse at 8-10 weeks old were housed under conditions of 12 hours light and 12 hours dark cycle (12:12). The mice were superovulated with $10 \mathrm{IU}$ pregnant mare serum gonadotropin (Cat no. 140825) through intraperitoneal injection. Mice were sacrificed by cervical dislocation after 46 hours. Immature oocytes were extracted from ovaries and collected in $\mathrm{M}_{2}$ medium (Cat no. M7167) under a stereo microscope. Immature oocytes at germinal vesicle (GV) stage were collected through dissecting method. The immature oocytes at GV stage divided into two parts that are, (1) denuded oocytes (DOs); (2) cumulus-oocyte complexes (COCs). The COCs were denuded of cumulus cells by adding $0.03 \%$ hyaluronidase (H3506, Sigma-Aldrich, St. Louis, MO, USA) and washed with $\mathrm{M}_{2}$ medium. The DOs were subjected to different concentrations of PTS $(0.1,0.25,0.5 \mu \mathrm{M})$ covered with mineral oil at $37{ }^{\circ} \mathrm{C}$ under $5 \% \mathrm{CO}_{2}$ in $95 \%$ humidified air. PTS at $0.25 \mu \mathrm{M}$ were used in further analysis. Group of 10 immature oocytes at GV stage were collected in three groups. In the first group with COCs was considered as a control in $25 \mu \mathrm{L}$ drops of the $\mathrm{M}_{2}$ medium. The DOs in the second and third group were added in $25 \mu \mathrm{M}$ of $\mathrm{M}_{2}$ media and $0.25 \mu \mathrm{M}$ PTS respectively. After 24 hours, oocytes were observed under an inverted microscope. In this study, IVM rate was determined by the appearance of the first polar body and were used for further analysis.

\subsection{Measurement of ROS and GSH Levels}

For detection of intracellular ROS and glutathione (GSH) level, 15-20 mature oocytes at M11 stage from each group was washed twice with polyvinyl alcohol (PVA) $(1 \mathrm{mg} / \mathrm{ml})$ and then incubated for 15 mins in $50 \mu \mathrm{L}$ of a droplet of $10 \mu \mathrm{M}$ 2, 7-dichlorodihydro-fluorescein (D6883, Sigma-Aldrich, St. Louis, MO, USA) and $10 \mu \mathrm{M}$ 4-chloromethyl-6, 8-difluoro-7-hydroxycoumarin (CMF2HC Cat. No. C12881 USA) respectively and put in incubator at $37{ }^{\circ} \mathrm{C}$ under $5 \% \mathrm{CO}_{2}$ in $95 \%$ humidified air. After incubation, the oocytes were washed twice with PBS and examined under an epifluorescent microscope (1X71 Olympus Tokyo, Japan). For ROS the emission and excitation wavelengths was $510 \mathrm{~nm}$ and $480 \mathrm{~nm}$ respectively. For GSH the emission and excitation 
wavelengths was $464 \mathrm{~nm}$ and $371 \mathrm{~nm}$ respectively. The data are analyzed in relative intensity of fluorescence. All pictures were taken under the same conditions and analyzed by Image-Pro plus 6.0 (Media Cybernetics, Rock ville, MD, USA).

\subsection{Western Blot Analysis}

Approximately 200 matured oocytes were selected from each group and lysed in a radioimmunoprecipitation assay (RIPA) (Beyotime, Jiangsu, China), while the nuclear and cytoplasmic proteins of oocytes were extracted with a nuclear protein extraction kit (Beyotime, China) for evaluation of NFE2L2 expression that contained a protease inhibitor and heated at $95^{\circ} \mathrm{C}$ for $5 \mathrm{~min}$. The total protein concentration in each group was detected on NANODROP one (Thermo Fischer Scientific). Protein lysate from oocytes was added to $12 \%$ sodium dodecyl sulfate-polyacrylamide gel (SDS-PAGE) electrophoresis. Moreover, the detached proteins were transferred to PVDF membranes (Millipore, USA). The membranes were blocked in skim milk for 1 hour and then membrane was incubated with primary antibodies KEAP 1 Rabbit Polyclonal Antibody (Protein Tech; Cat no. 10503-2-AP), NFE2L2 Rabbit Polyclonal Antibody (Protein Tech; Cat no. 16396-1-AP), BCL2 Rabbit Polyclonal Antibody (Protein Tech; Cat no. 12789-1-AP), BAX Rabbit Polyclonal Antibody (Protein Tech; Cat no. 50599-2-Ig) and GADPH antibody (Rabbit Polyclonal Antibody (Protein Tech; Cat no. 104944-1-AP) at $4{ }^{\circ} \mathrm{C}$ overnight. Afterward, the membrane in TBST was washed thrice times and was incubated at room temperature for 2 hours with Peroxidase Conjugated Affinipu Goat Anti Rabbit IgG (HL) (Protein Tech; Cat no. SA 00001-2). The target protein bands were detected using a chemiluminescence Plus reagent (ECL Plus, Bio sharp Life Science, cat no. BL50A).

\subsection{RNA Isolation, cDNA Synthesis, and Semiquantitative Real-Time Polymerase Chain Reaction (RT-PCR)}

For RNA isolation three biological replicates from each treated group were used. Total RNA was isolated from 150 matured oocytes by using an RNA isolation kit (Qiagen RNeasy Mini Kit Cat no. 74104) according to the manufacturer's instruction. cDNA was created by reverse transcription using Prime Script ${ }^{\mathrm{TM}}$ RT reagent Kit with gDNA Eraser (Takara Biotechnology Co. Ltd., Dalian, China Cat no. PR047A). The concentration of total RNA was measured NANODROP one (Thermo Fischer Scientific). The PCR was done by using $25 \mu \mathrm{L}$ reaction volumes including $4 \mu \mathrm{L}$ cDNA, $6 \mu \mathrm{L} \mathrm{H}_{2} \mathrm{O}, 1 \mu \mathrm{L}$ reverse primers, $1 \mu \mathrm{L}$ forward primers $(5 \mathrm{mM})$ and $13 \mu \mathrm{L} 2 \times \mathrm{Taq}$ PCR Master Mix (Tiangen Biotech Co., Ltd. Cat no. KT201). The condition for PCR are as follow, $95^{\circ} \mathrm{C}$ for 3 mins initial pre-incubation level, then $95^{\circ} \mathrm{C}$ for 30 secs denaturation, $59-62{ }^{\circ} \mathrm{C}$ for 30 secs annealing, $72{ }^{\circ} \mathrm{C}$ for 30 secs extension for 35 cycles and $72{ }^{\circ} \mathrm{C}$ for $5 \mathrm{mins}$ in final extension. Primer sequence and annealing temperature are listed in (Table 1). The bands for the genes were analyzed on on $2 \%$ gels by electrophoresis having ethidium bromide. The bands were visualized under UV illumination and intensities were analyzed by Lane 1D Analysis Software (Beijing Sage Creation Science Co., Ltd., Beijing, China).

Table 1. Primer sequences

\begin{tabular}{llllll}
\hline Gene & Access No. & Forward primer (5'-3') & Reverse primer (5'-3') & Tm $\left({ }^{\circ} \mathbf{C}\right)$ & Amplicon size, bp \\
\hline$G A P D H$ & BC023196 & CATCACCATCTTCCAGGAGCG & GAGGGGCCATCCACAGTCTTC & 59 & 357 \\
$H M O X 1$ & NM010442 & CAGGTGATGCTGACAGAGGA & ACAGGAAGCTGAGAGTGAGG & 62 & 184 \\
$C A T$ & NM009804 & GGAGGCGGGAACCCAATAG & GTGTGCCATCTCGTCAGTGAA & 59 & 102 \\
$S O D 1$ & NM011434 & TTCGAGCAGAAGGCAAGCGGTGAA & AATCCCAATCACACCACAAGCCAA & 59 & 396 \\
$B A X$ & NM007527 & CCAGGATGCGTCCACCAA & AAGTAGAAGAGGGCAACCAC & 62 & 195 \\
$B C L 2$ & NM007527 & AACTCTTCAGGGATGGGG & GCCGGTTCAGGTACTCAG & 59 & 144 \\
\hline
\end{tabular}

\subsection{Statistical Analysis}

Unless otherwise indicated, data were presented in mean \pm standard deviations. All the data were calculated by using multiple comparisons (IBM SPSS 17.0) and one-way ANOVA followed by post-hoc Turkey's multiple comparison tests (Graph Pad Prism 5 software). Statistical significance was considered at $(P<0.05)$ and data were performed in three separate events.

\section{Results}

\subsection{The Effect of Different Concentration of Pterostilbene on the Nuclear Maturation of Mouse Oocytes}

In our initial experiment, the $\mathrm{M}_{2}$ IVM media of DOs was supplemented with different concentrations of PTS $(0.1$, 0.25 and $0.5 \mu \mathrm{M})$. PTS at a $0.25 \mu \mathrm{M}$ concentration significantly increased the percentage $(P<0.05)$ of M11 stage 
DOs compared to DOs treated group (Table 2). So, for further analysis, $0.25 \mu \mathrm{M}$ of PTS was used in the different experiment.

Table 2. The effect of different concentration of PTS on the nuclear maturation of Mouse oocytes

\begin{tabular}{llll}
\hline Group & Total No. of GV Oocyte $\uparrow$ & Total No. of GVBD (\%) & Total No. of Maturation MII (\%) \\
\hline DOs & 213 & $72.0 \pm 1.85 \mathrm{~b}$ & $52.1 \pm 1.60 \mathrm{~b}$ \\
DOs $+0.1 \mu \mathrm{M}$ PTS & 208 & $81.7 \pm 1.79 \mathrm{a}$ & $68.7 \pm 1.16 \mathrm{a}$ \\
DOs $+0.25 \mu$ M PTS & 207 & $83.9 \pm 2.12 \mathrm{a}$ & $70.4 \pm 1.55 \mathrm{a}$ \\
DOs $+0.5 \mu$ M PTS & 205 & $79.5 \pm 3.94 \mathrm{a}$ & $66.8 \pm 2.30 \mathrm{a}$ \\
\hline
\end{tabular}

Note. ab: Values within a column with different superscripts significantly differ $(P<0.05)$. $\dagger$ : Experiment was replicated more than 5 times.

\subsection{The Effect of Pterostilbene on the Developmental Potential in MII Oocytes}

As shown in Table 3, the MII rate of the DOs + PTS group (57.6 $\pm 1.77 \%)$ was significantly higher than the DOs group $(42.6 \pm 1.92 \% ; P<0.05)$ but similar to the COCs group $(67.1 \pm 2.24 \% ; P>0.05)$.

Table 3. Effect of $0.25 \mu \mathrm{M}$ pterostilbene on mouse oocytes maturation

\begin{tabular}{llll}
\hline Group & Total No. of GV oocyte $\dagger$ & Total No. of GVBD (\%) & Total No. of Maturation MII (\%) \\
\hline COCs & 212 & $86.3 \pm 1.06 \mathrm{a}$ & $75.6 \pm 1.71 \mathrm{a}$ \\
DOs & 213 & $72.0 \pm 1.85 \mathrm{~b}$ & $52.1 \pm 1.60 \mathrm{~b}$ \\
DOs $+0.25 \mu$ M PTS & 207 & $83.9 \pm 2.12 \mathrm{a}$ & $70.4 \pm 1.55 \mathrm{a}$ \\
\hline
\end{tabular}

Note. ab: Values within a column with different superscripts significantly differ $(P<0.05) . \dagger$ : Experiment was replicated more than 5 times.

\subsection{Effect of Pterostilbene on Intracellular ROS and GSH Levels}

To determine the intracellular level of ROS and GSH, M11 stage oocytes were collected and stained with DCHFDA (Figure 1A) and CMF2HC (Figure 1A). The ROS level in DOs group was significantly higher $(P<$ 0.05 ) in the M11 stage as compared to the COCs and those treated with DOs + PTS (Figure 1B). By contrast, GSH level in DOs group was significantly lower $(P<0.05)$ in M11 stage compared to the COCs and those treated with DOs + PTS (Figure 1B). 
(A)

$\operatorname{cocs}$
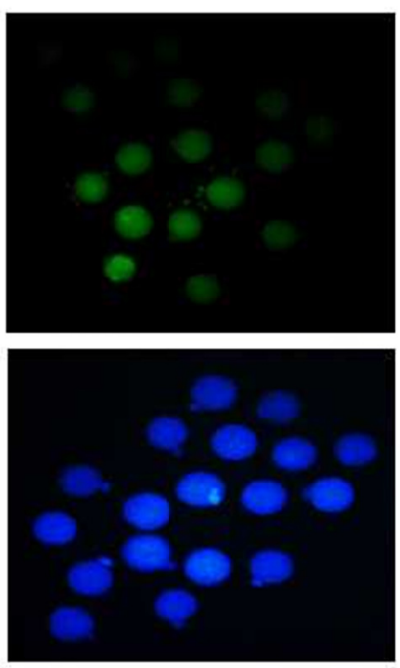

DOs
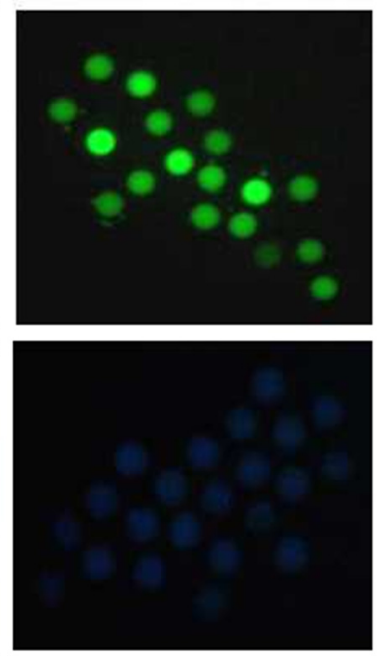

DOs + PTS
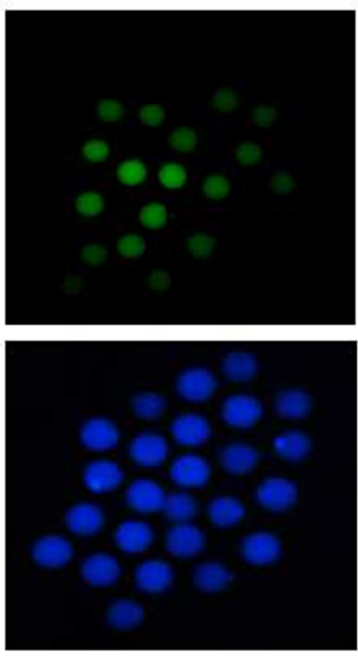

ROS

GSH

(B)
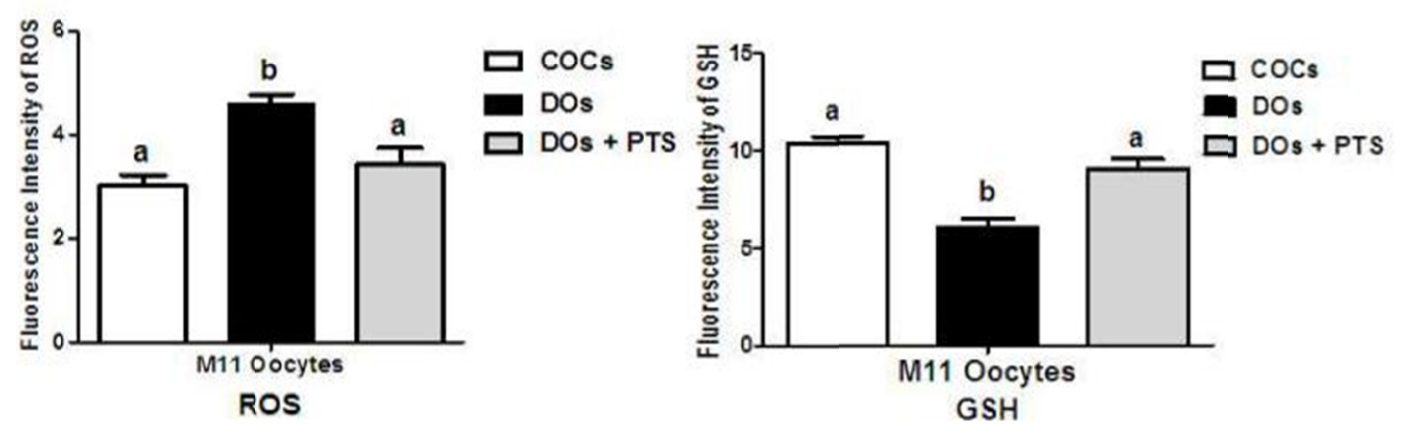

Figure 1. ROS and GSH content in M11 stage oocytes

Note. (A) Fluorescence microscopy of the oocytes matured in different cultured media by staining with DCHFDA and CMF2HC respectively. Images are presented at X400 magnification. (B) Fluorescence intensity was quantified by Image-Pro Plus 6.0. Different letter ab Values were significantly different $(P<0.05)$.

\subsection{Effect of Pterostilbene on Nuclear Translocation of NFE2L2 in the Mouse Oocytes}

NFE2L2 activators induce separation of the NFE2L2-KEAP1 complex in the cytoplasm, thus its lead NFE2L2 to translocation into the nucleus to bind with ARE-binding protein to activate the cellular protective gene expression (Oh \& Jun, 2018). To identify whether the PTS translocate the NFE2L2, its concentrations were measured in the cytoplasmic and nuclear extracts of PTS pretreated in mouse M11 stage oocytes. DOs + PTS treated group increased NFE2L2 expression in the nuclear extracts was significantly higher $(P<0.05)$ compared to DOs group (Figures 2A and 2C). However, the protein level of NFE2L2 was decreased in cytoplasmic extracts as compared to the DOs treated group in M11 stage mouse oocytes (Figures 2A and 2D). Furthermore, the KEAP1 protein level in DOs + PTS treated group was significantly lowered $(P<0.05)$ compared to DOs group (Figures $2 \mathrm{~A}$ and $2 \mathrm{~B}$ ). These results provide a strong evidence that PTS induces NFE2L2 activation and its nuclear translocation in the mouse oocytes. 
(A)

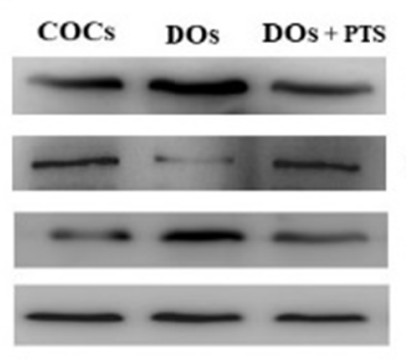

(C)

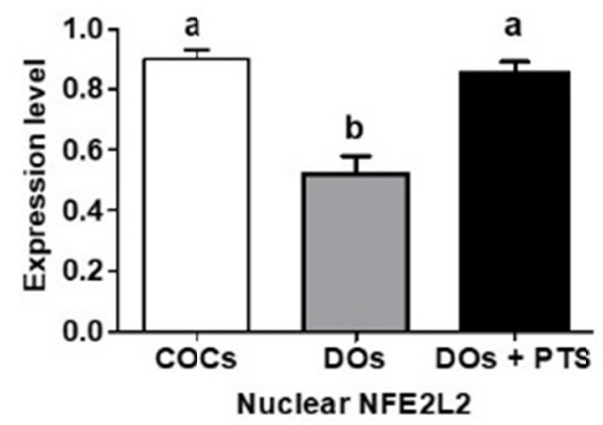

(B)

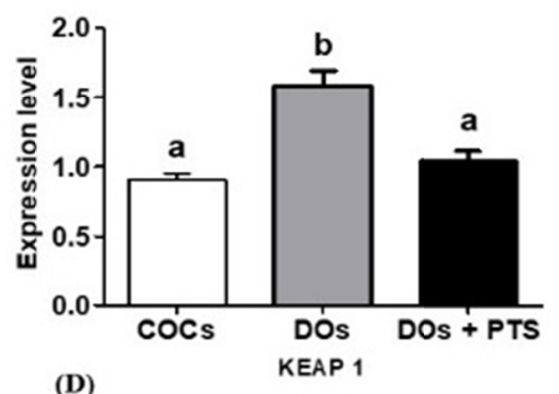

(D)

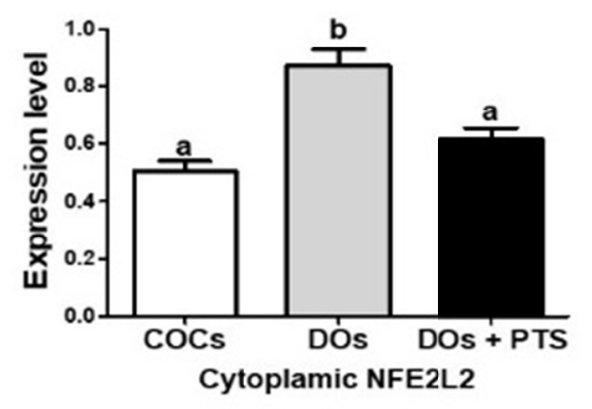

Figure 2. Effect of PTS on NFE2L2 and KEAP1 protein expression in mouse M11 stage oocytes matured in the different groups

Note. (A) Western Blot results of KEAP1, Cytoplasmic and Nuclear NFE2L2 protein expression level in the mouse oocytes. (B) Relevant expression level of KEAP1 (C) Nuclear (D) Cytoplasmic NFE2L2 with GADPH as an internal marker $(P<0.05)$. Different letters ab Values were significantly different $(P<0.05)$.

\subsection{Effect of Pterostilbene on NFE2L2 Downstream Target Genes Expression}

We analyzed PTS effect on antioxidant enzymes downstream of NFE2L2 activation and nuclear translocation. DOs + PTS treatment group significantly increased the expression of HMOX1, CAT and SOD (Figures 3A and 3B) compared with the DOs group. These results suggest that PTS enhances the expression of antioxidant genes triggered by NFE2L2, thereby protecting mouse oocytes during in vitro maturation.

3.6 Effect of Pterostilbene on Apoptosis Gene in the Mouse Matured Oocytes

The effect of PTS treatment on apoptotic-related gene expression in M11 stage mouse oocytes has been shown in Figure 3. PTS significantly up-regulated the RNA expression of an anti-apoptotic gene, BCL2 (Figures 3A and $3 \mathrm{~B})$ with a concomitant reduction in the expression of proapoptotic BAX compared to DOs treated group (Figures $3 \mathrm{~A}$ and $3 \mathrm{~B}$ ). 


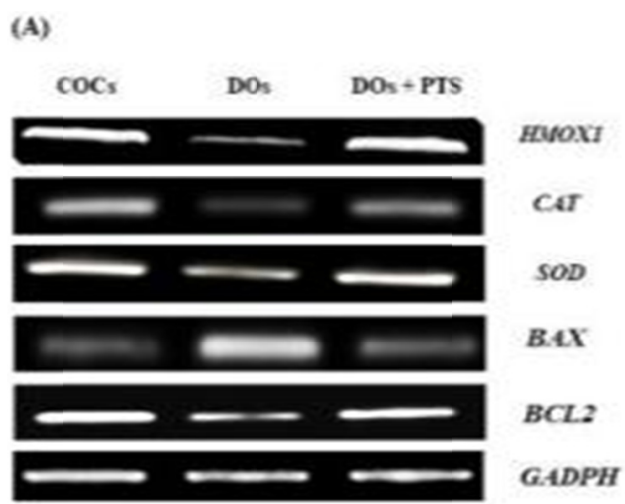

(B)
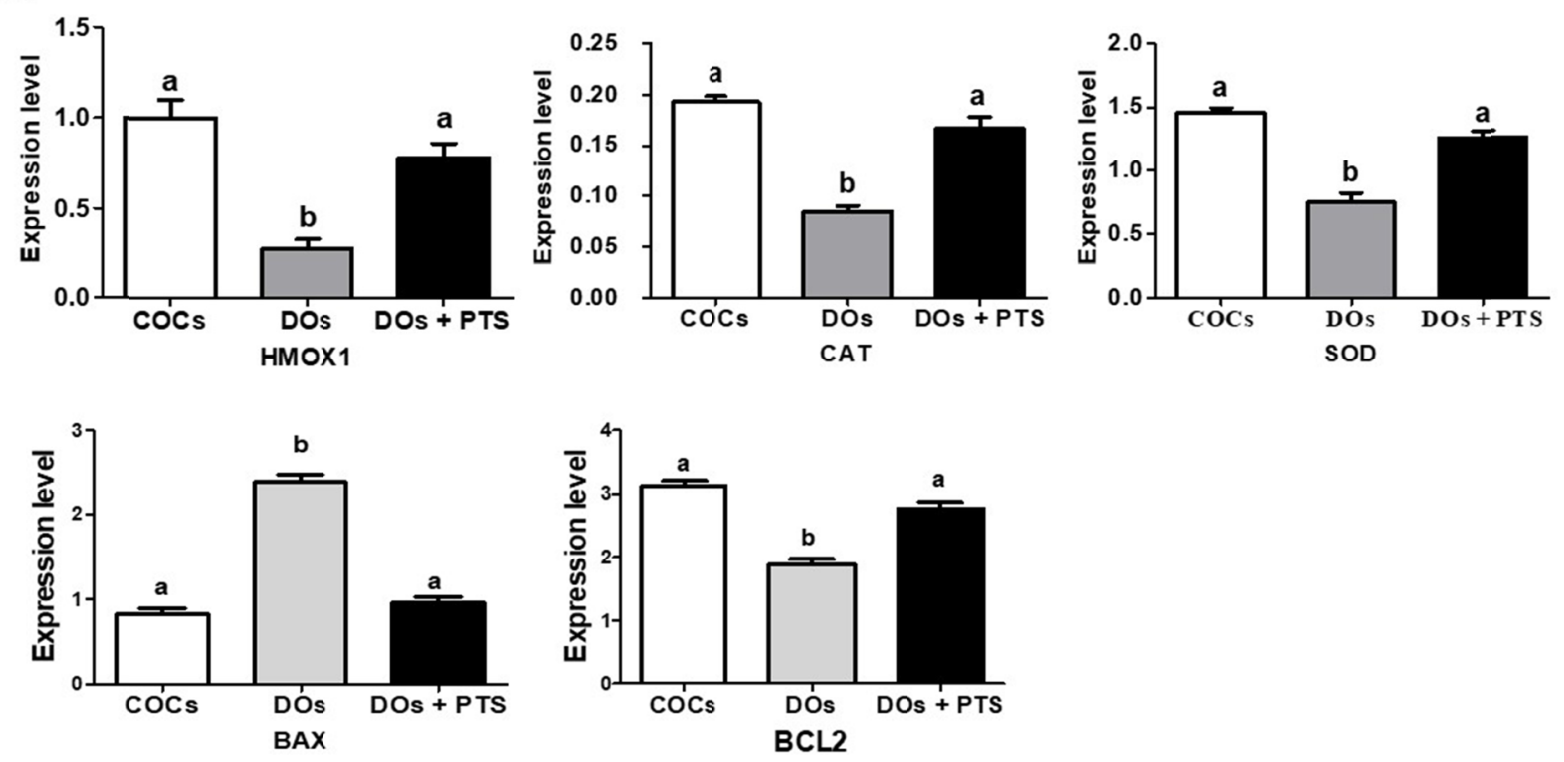

Figure 3. Effect of pterostilbene on antioxidant enzymes and apoptotic gene expression

Note. (A) PCR results of HMOX1, CAT, SOD, BCL2, and BAX expression in mouse M11 stage oocytes matured in the different groups. (B) Relative changes of HMOX1, CAT, SOD, BCL2, and BAX with GADPH as an internal marker $(P<0.05)$. Different letter ab Values were significantly different $(P<0.05)$.

\subsection{Effect of Pterostilbene on Apoptosis Protein Expression Levels in the Mouse Matured Oocytes}

Apoptosis induced by ROS can trigger oxidation in the mouse oocytes (Kim, Jeon, Kim, Lee, \& Hyun, 2015). So, we analyzed the effect of PTS on the expression of pro-apoptotic proteins BAX and antiapoptotic protein BCL2. PTS treatment significantly increased the expression of antiapoptotic protein BCL2 compared to DOs group (Figure 4B), but significantly decreased the expression level of proapoptotic protein BAX compared to DOs group (Figure 4A). 
(A)
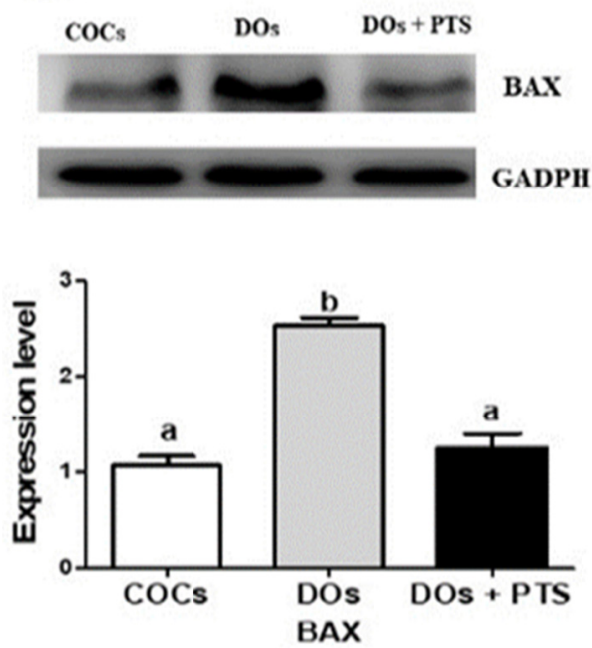

(B)
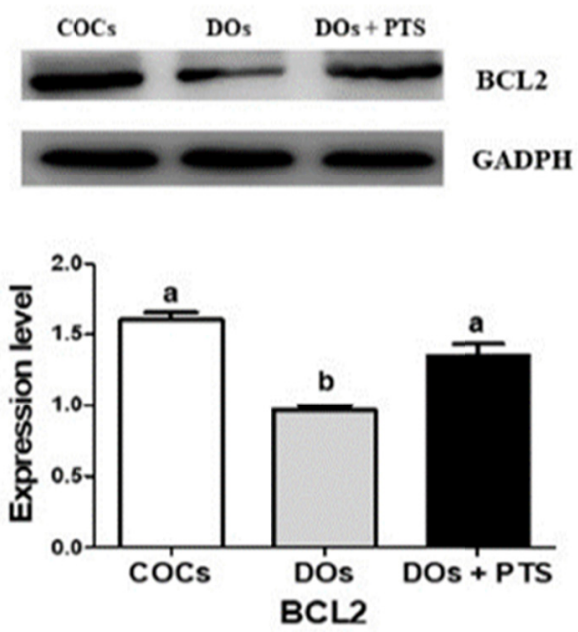

Figure 4. Effect of pterostilbene treatments on apoptotic protein expression

Note. (A, B) Western blot results of BCL2 and BAX expression in control and treated groups in M11 stage oocytes matured. (A, B) Relative changes of BCL2 and BAX with GADPH as an internal marker $(P<0.05)$. Different letter ab Values were significantly different $(P<0.05)$.

\section{Discussion}

In mammalian species, cumulus cells have an important role during oocytes maturation and development, as it associated with oocytes by gap junction communication (de Matos \& Furnus, 2000). Glutathione (GSH) synthesis is regulated by cumulus cells (de Matos \& Furnus, 2000), to retain the redox state in cells and protect the cell from oxidative injuries (Gasparrini et al., 2006). Previous studies highlighted that natural antioxidant through in vitro maturation media protects the oocytes from oxidative stress (Golkar-Narenji et al., 2010; Wang et al., 2007). Recently epigallocatechin-3-gallate a natural polyphenol has been given in bovine in vitro oocytes maturation and has improved oocytes maturation (Huang et al., 2018). PTS also is a natural polyphenol compound, as its play momentous role in the protection against oxidative injuries in the cell (Wang et al., 2016). In this study, we used PTS for the first time in immature DOs. Different concentration of PTS $(0.1,0.25$, and 0.5 $\mu \mathrm{M})$ was added to mouse maturation media in DOs, the $0.25 \mu \mathrm{M}$ of PTS increased the maturation rate of M11 stage oocytes in mouse compared to other groups (Table 2). Therefore, PTS at $0.25 \mu \mathrm{M}$ were used for further analysis. Our study revealed that PTS increased the maturation rate of mouse oocytes in DOs, as shown in other studies that melatonin increased the IVM quality in DOs as well as significantly lowered the ROS level in bovine (Zhao et al., 2015). By adding, PTS in $\mathrm{DO}_{\mathrm{S}}$ it significantly lowers the ROS levels (Figures 1A and 1B). By contrast, PTS increased the GSH level in DOs (Figures 1A and 1B). Previous studies evaluated that GSH is lower in DOs compared to COs enclosed oocytes in Cattle (de Matos \& Furnus, 2000; Zhao et al., 2015) and Pig (Cui et al., 2009).

In numerous studies, the activation of NFE2L2-KEAP1 pathway displays a defensive role to counter oxidative stress injuries during inflammation, diabetes, cancer, neurodegenerative disorders (Chang et al., 2012; Xue et al., 2017). The activation of the NFE2L2-KEAP1 pathway has been distinguished to have a positive effect in oocytes maturation to counter oxidative stress by regulating several antioxidants and cytoprotective genes that extended cell viability (Kim et al., 2015). Recently Lin et al. (2018) confirmed that inhibition of NFE2L2 by brusatol in mice oocytes can disturb the proper spindle and chromosome formation in meiotic oocytes. PTS has been found to activate the NFE2L2 mediated signaling pathway in pancreatic beta-cell and exerted upregulation of NFE2L2 downstream antioxidant enzymes, such as HMOX1, GPX, SOD, and CAT (Bhakkiyalakshmi et al., 2014). Our western blot analysis data also recognized that addition of PTS to DOs in maturation media exerted the NFE2L2 translocation from cytoplasm to the nucleus and lowering the KEAP1 protein expression levels. Moreover, our data also found that PTS increased the expression level of HMOXI, CAT, and SOD.

During maturation of oocytes, ROS are responsible in the in vitro condition for the initiation of apoptosis (Kim et al., 2015). During intrinsic stress, the stress condition can cause the induction of apoptosis in mice oocytes maturation (Zhao et al., 2015) through the activation of proapoptotic BAX which leads to cytochrome C release 
from the mitochondria, resulting in selective apoptosis (Sireesh et al., 2017). However, treatment with an antioxidant can protect the oocytes from oxidative stress damage by regulation of BCL2 family (Kim et al., 2015). PTS addition in DOs maturation media in our result have shown down-regulation in the expression level of pro-apoptotic genes BAX but on another side, PTS increased the expression level of anti-apoptotic gene BCL2. Western Blot analysis of BAX protein showed the down-regulation by PTS and up-regulation of BCL2 in DOs confirms the protective effect of PTS. A similar study was reported in induce diabetic animals, as it improved the BCL2 and lower BAX expression by activation of NFE2L2 by PTS in pancreatic beta-cells against different insult such as oxidative stress (Sireesh et al., 2017). All these results predicted that activation of an NFE2L2 signaling pathway by PTS can improve oocytes maturation rate in the mouse.

\section{Conclusions}

In conclusion, our data highlighted that PTS can improve the nuclear maturation of mouse DOs, enhanced survival of DOs via NFE2L2 signaling pathway, evaluate the ROS and GSH levels and reduce the apoptotic rates of DOs in absence of cumulus cell. These results identifying the potential role of PTS as an antioxidant in oocytes maturation.

\section{References}

Alessandro, M., Marco, S., di Osti, F., \& Cesari, A. (2000). Bioassays on the Activity of Resveratrol, Pterostilbene and Phosphorous Acid towards Fungi Associated with Esca of Grapevine. Phytopathologia Mediterranea, 39(3), 357-365. https://doi.org/10.14601/Phytopathol_Mediterr-1574

Amin, A., Gad, A., Salilew-Wondim, D., Prastowo, S., Held, E., Hoelker, M., ... Tesfaye, D. (2014). Bovine embryo survival under oxidative-stress conditions is associated with activity of the NFE2L2-mediated oxidative-stress-response pathway. Molecular Reproduction and Development, 81(6), 497-513. https://doi.org/10.1002/mrd.22316

Bhakkiyalakshmi, E., Shalini, D., Sekar, T. V., Rajaguru, P., Paulmurugan, R., \& Ramkumar, K. M. (2014). Therapeutic potential of pterostilbene against pancreatic beta-cell apoptosis mediated through NFE2L2. British Journal of Pharmacology, 171(7), 1747-1757. https://doi.org/10.1111/bph.12577

Chang, J., Rimando, A., Pallas, M., Camins, A., Porquet, D., Reeves, J., ... Casadesus, G. (2012). Low-dose pterostilbene, but not resveratrol, is a potent neuromodulator in aging and Alzheimer's disease. Neurobiology of Aging, 33(9), 2062-2071. https://doi.org/10.1016/j.neurobiolaging.2011.08.015

Chiou, Y. S., Tsai, M. L., Nagabhushanam, K., Wang, Y. J., Wu, C. H., Ho, C. T., \& Pan, M. H. (2011). Pterostilbene is more potent than resveratrol in preventing azoxymethane (AOM)-induced colon tumorigenesis via activation of the NF-E2-related factor 2 (NFE2L2)-mediated antioxidant signaling pathway. Journal of Agricultural and Food Chemistry, 59(6), 2725-2733. https://doi.org/10.1021/jf2000103

Cui, M. S., Fan, Y. P., Wu, Y., Hao, Z. D., Liu, S., Chen, X. J., \& Zeng, S. M. (2009). Porcine cumulus cell influences ooplasmic mitochondria-lipid distributions, GSH-ATP contents and calcium release pattern after electro activation. Theriogenology, 71(3), 412-421. https://doi.org/10.1016/j.theriogenology.2008.08.008

de Matos, D. G., \& Furnus, C. C. (2000). The importance of having high glutathione (GSH) level after bovine in vitro maturation on embryo development: Effect of $\beta$-mercaptoethanol, cysteine, and cystine. Theriogenology, 53(3), 761-771. https://doi.org/10.1016/S0093-691X(99)00278-2

Gad, A., Hoelker, M., Besenfelder, U., Havlicek, V., Cinar, U., Rings, F., ... Tesfaye, D. (2012). Molecular mechanisms and pathways involved in bovine embryonic genome activation and their regulation by alternative in vivo and in vitro culture conditions. Biology of Reproduction, 87(4), 100. https://doi.org/ 10.1095/biolreprod.112.099697

Gasparrini, B., Boccia, L., Marchandise, J., Di Palo, R., George, F., Donnay, I., \& Zicarelli, L. (2006). Enrichment of in vitro maturation medium for buffalo (Bubalus bubalis) oocytes with thiol compounds: Effects of cystine on glutathione synthesis and embryo development. Theriogenology, 65(2), 275-287. https://doi.org/10.1016/j.theriogenology.2005.05.036

Golkar-Narenji, A., Eimani, H., Samadi, F., Hasani, S., Shahverdi, A, H., Eftekhari-Yazdi, P., \& Kamalinejad, M. (2010). Effect of Papaver rhoeas extract on in vitro maturation and developmental competence of immature mouse oocytes. Reproductive Medicine and Biology, 9(4), 211-215. https://doi.org/10.1007/s12522-0100059-0 
Harris, C., \& Hansen, J. M. (2012). Nrf2-mediated resistance to oxidant-induced redox disruption in embryos. Birth Defects Research, Part B, Developmental and Reproductive Toxicology, 95(3), 213-218. https://doi.org/10.1002/bdrb.21005

Huang, Z., Pang, Y., Hao, H., Du, W., Zhao, X., \& Zhu, H. (2018). Effects of epigallocatechin-3-gallate on bovine oocytes matured in vitro. Asian-Australasian Journal of Animal Sciences. https://oi.org/10.5713/ ajas. 17.0880

Jiang, T., Huang, Z., Lin, Y., Zhang, Z., Fang, D., \& Zhang, D. D. (2010). The protective role of NFE2L2 in streptozotocin-induced diabetic nephropathy. Diabetes, 59(4), 850-860. https://doi.org/10.2337/db09-1342

Kasiotis, K. M., Pratsinis, H., Kletsas, D., \& Haroutounian, S. A. (2013). Resveratrol and related stilbenes: Their anti-aging and anti-angiogenic properties. Food and Chemical Toxicology, 61, 112-120. https://doi.org/ 10.1016/j.fct.2013.03.038

Kim, E., Jeon, Y., Kim, D. Y., Lee, E., \& Hyun, S. H. (2015). Antioxidative effect of carboxyethyl germanium sesquioxide (Ge-132) on IVM of porcine oocytes and subsequent embryonic development after parthenogenetic activation and IVF. Theriogenology, 84(2), 226-36. https://doi.org/10.1016/j.theriogenology. 2015.03.006

Lee, H. H., Park, S. A., Almazari, I., Kim, E. H., Na, H. K., \& Surh, Y. J. (2010). Piceatannol induces heme oxygenase-1 expression in human mammary epithelial cells through activation of ARE-driven NFE2L2 signaling. Archives of Biochemistry and Biophysics, 501(1), 142-150. https://doi.org/10.1016/j.abb. 2010.06.011

Lin, Y., Sui, L. C., Wu, R. H., Ma, R. J., Fu, H. Y., Xu, J. J., ... Chen, L. (2018). Nrf2 inhibition affects cell cycle progression during early mouse embryo development. Journal of Reproduction and Development, 64(1), 49-55. https://doi.org/10.1262/jrd.2017-042

Ma, R., Li, H., Zhang, Y., Lin, Y., Lin, Y., Qiu, X., Xie, M., \& Yao, B. (2017). The toxic effects and possible mechanisms of Brusatol on mouse oocytes. PLoS One, 12(5), e0177844. https://doi.org/10.1371/ journal.pone.0177844

Mehaisen, G. M., Saeed, A. M., Gad, A., Abass, A. O., Arafa, M., \& El-Sayed, A. (2015). Antioxidant Capacity of Melatonin on Preimplantation Development of Fresh and Vitrified Rabbit Embryos: Morphological and Molecular Aspects. PLoS One, 10(10), e0139814. https://doi.org/10.1371/journal.pone.0139814

Na, H. K., Kim, E. H., Jung, H., Lee, H. H., Hyun, J. W., \& Surh, Y. J. (2008). (-)-Epigallocatechin gallate induces NFE2L2-mediated antioxidant enzyme expression via activation of PI3K and ERK in human mammary epithelial cells. Archives of Biochemistry and Biophysics, 476(2), 171-177. https://doi.org/ 10.1016/j.abb.2008.04.003

Oh, Y. S., \& Jun, H. S. (2018). Effects of Glucagon-Like Peptide-1 on Oxidative Stress and NFE2L2 Signaling. International Journal of Molecular Sciences, 19(1), 26. https://doi.org/10.3390/ijms19010026

Rossi, D., Guerrini, A., Bruni, R., Brognara, E., Borgatti, M., Gambari, R., ... Sacchetti, G. (2012). trans-Resveratrol in nutraceuticals: issues in retail quality and effectiveness. Molecules, 17(10), 12393-12405. https://doi.org/10.3390/molecules

Sireesh, D., Ganesh, M. R., Dhamodharan, U., Sakthivadivel, M., Sivasubramanian, S., Gunasekaran, P., \& Ramkumar, K. M. (2017). Role of pterostilbene in attenuating immune mediated devastation of pancreatic beta cells via NFE2L2 signaling cascade. The Journal of Nutritional Biochemistry, 44, 11-21. https://doi.org/10.1016/j.jnutbio.2017.02.015

Stivala, L. A., Savio, M., Carafoli, F., Perucca, P., Bianchi, L., Maga, G., ... Vannini, V. (2001). Specific structural determinants are responsible for the antioxidant activity and the cell cycle effects of resveratrol. The Journal of Biological Chemistry, 276(25), 22586-22594. https://doi.org/10.1074/jbc.M101846200

Ungvari, Z., Bagi, Z., Feher, A., Recchia, F. A., Sonntag, W. E., Pearson, K., ... Csiszar, A. (2010). Resveratrol confers endothelial protection via activation of the antioxidant transcription factor NFE2L2. American Journal of Physiology-Heart and Circulatory Physiology, 299(1), 18-24. https://doi.org/10.1152/ajpheart. 00260.2010

Wang, B., Liu, H., Yue, L., Li, X., Zhao, L., Yang, X., ... Qu, Y. (2016). Neuroprotective effects of ptrostilbene against oxidative stress injury: Involment of nuclear factor erthroid 2-related factor 2 pathway. Brain Research, 1643, 70-79. https://doi.org/10.1016/j.brainres.2016.04.048 
Wang, Z., Yu, S., \& Xu, Z. (2007). Effect of supplementation of green tea polyphenols on the developmental competence of bovine oocytes in vitro. Brazilian Journal of Medical and Biological Research, 40(8), 1079-85. https://doi.org/10.1590/S0100-879X2007000800008

Xue, E. X., Lin, J. P., Zhang, Y., Sheng, S. R., Liu, H. X., Zhou, Y. L., \& Xu, H. (2017). Pterostilbene inhibits inflammation and ROS production in chondrocytes by activating NFE2L2 pathway. Oncotarget, 8(26) 41988-42000. https://doi.org/10.18632/oncotarget.16716

Zhao, X. M., Min, J. T., Du, W. H., Hao, H. S., Liu, Y., Qin, T., ... Zhu, H. B. (2015). Melatonin enhances the in vitro maturation and developmental potential of bovine oocytes denuded of the cumulus oophorus. Zygote, 23(4), 525-36. https://doi.org/10.1017/S0967199414000161

\section{Copyrights}

Copyright for this article is retained by the author(s), with first publication rights granted to the journal.

This is an open-access article distributed under the terms and conditions of the Creative Commons Attribution license (http://creativecommons.org/licenses/by/4.0/). 